\title{
SALIVARY GLAND ONCOCYTOMA AND EVOLUTION OF ONCOCYTES
}

\author{
Reshma Amin 1 , Pushparaja Shetty²
}

${ }_{1}^{1}$ Lecturer, Department of Oral Pathology and Microbiology, AB Shetty Institute of Dental Sciences, Deralakatte, Mangalore, Karnataka, India.

2Professor and HOD, Department of Oral Pathology and Microbiology, AB Shetty Institute of Dental Sciences, Deralakatte, Mangalore, Karnataka, India.

\begin{abstract}
BACKGROUND
ABSTRACT

Various glandular and secretory epithelia show oncocytes. Most of the oncocytic lesions are benign with a relatively low rate of proliferation. Oncocytomas form less than $1 \%$ of all benign salivary gland tumours, $82 \%$ to $90 \%$ of them occur in the parotid gland, sometimes in the submandibular gland (9\%) and uncommon in the intraoral minor salivary glands. The oncocytomas occur in adults in their sixth to eighth decades, with no sex predilection. The morphological and functional alteration in unusual oncocytes is due to a compensatory mechanism to overcome energy deficient state in the cell undergoing changes. Several theories on biochemical changes occurring in mitochondria explain the formation of these oncocytes. The mitochondrial DNA point mutations due to altered oxidative phosphorylation complexes are one of the main downsides for the oncocytic genesis. The hypothesis of their origin of transformation has been put forward by several researchers. The role of the numerous mitochondria and their functional inability to maintain the bioenergetics pathway forms the basis for these oncocytic changes.
\end{abstract}

\section{KEY WORDS}

Oncocytes, Salivary glands, Aetiology, Biochemical Change, Mitochondrial DNA

HOW TO CITE THIS ARTICLE: Amin R, Shetty P. Salivary gland oncocytoma and evolution of oncocytes. J. Evolution Med. Dent. Sci. 2019;8(14):1196-1198, DOI: 10.14260/jemds/2019/263

\section{BACKGROUND}

Various glandular and secretory epithelia show oncocytes. Most of the oncocytic lesions are benign with a relatively low rate of proliferation. These eosinophilic modified cells are prevalent among salivary glands, buccal mucosa, pituitary, thyroid, parathyroid, nasal cavities, sinuses, ocular caruncle, lacrimal glands, Eustachian tube and the larynx in the head and neck region. They are indistinguishable from each other throughout the various anatomic sites. Tandler et al. in 1964 revealed with electron microscopy that oncocytes contained unusually high numbers of mitochondria. Oncocytic lesions in salivary glands were found to be consisting of swollen granular cells in the ductal and acinar elements. Oncocytomas form less than $1 \%$ of all benign salivary gland tumours, $82 \%$ to $90 \%$ of them occur in the parotid gland, at times affect the submandibular gland (9\%) and uncommon in the intraoral minor salivary glands. They are generally unilateral, sometimes bilateral oncocytomas have been reported. The oncocytomas occur in adults in their sixth to eighth decades, with no sex predilection. ${ }^{1-6}$ The morphological and functional alteration in unusual oncocyte is due to a compensatory mechanism to overcome energy deficient state in the cell undergoing changes. The eosinophilic oncocytes represent part of senescent degeneration, few epithelial cells of salivary glands are the targets of these oncocytic changes. The primary element of metaplasia is the functional enzymes involved in the cellular biochemical pathway, and it has been a center of interest in many studies.

'Financial or Other Competing Interest': None.

Submission 01-02-2019, Peer Review 26-03-2019,

Acceptance 01-04-2019, Published 08-04-2019.

Corresponding Author:

Dr. Reshma Amin

Department of Oral Pathology and Microbiology,

A B Shetty Institute of Dental Sciences,

Deralakatte-575018, Mangalore, Karnataka, India.

E-mail: reshyesh@yahoo.com

DOI: $10.14260 /$ jemds $/ 2019 / 263$
These changes of oncocytic transformation in various organs is age-dependent functional impairment. But a homoplasmic mutation in the mitochondrial DNA (mtDNA) has also been reported.7,8,9,10

This article reviews the overall changes observed in oncocytic metaplasia of the salivary gland with histopathologic findings emphasizing on the origin of oncocytes in general.

\section{Aetiology and Theories of Origin}

The World Health Organization classifies the oncocytic neoplasms of salivary gland tumours into three categories: nodular oncocytic hyperplasia, oncocytoma, and oncocytic carcinoma. The presence of oncocytes in salivary gland ductal epithelium is rare before the age of 50. As age advances alterations in structure and function of glandular, ductal structures are typical. Though there are no certain etiologic factors mtDNA mutations sometimes are associated with radiation, ultraviolet light, ozone, metals, pesticides, air pollutants, pharmaceutical drugs, asbestos, and arsenic. Oncocytomas originate mainly from the intercalated duct cells consequently most of the oncocytomas occur in the parotid gland abundant in intercalated ducts. Several theories on biochemical changes occurring in mitochondria explain about the formation of these oncocytes. During the life cycle, cytoplasmic segregation occurs, giving rise to a range of cells varying in bioenergetic capacity. Mitochondrial mutation sometimes can happen due to a constant source of irritation. Thus, cells that accumulate large numbers of mitochondrial mutations will eventually get affected. Mitochondrial oxidative metabolism increases under certain stress conditions and such energy demands lead to a clinical manifestation detectable in the different gland types. With age, few acinar cells get replaced by fat and connective tissues, which are incapable of secreting fluid. In adult lifespan, these changes happen, but functional levels of gland in general remain. Interaction of advanced glycation end products with cellular receptors alters the level of gene expression. The cells with 
altered gene expression can undergo apoptosis, oxidative stress, cytotoxicity, and necrosis. This scenario may not be caused by a single stimulus alone since advanced glycated end products are inducible by oxidative mechanisms as well as oxidative stress. ${ }^{9}$ Mitochondria number raises due to a functional defect; there is minimal production of adenosine triphosphate constitutes the basis for understanding the development of oncocytic neoplasms and their trend towards multicentricity. ${ }^{5-14}$

It is evident that the oncocytes increase with age. The mitochondrial DNA can be the target to stress from multiple factors. The changes accumulate in a long span of life leads to reduced function of the power house of the cell. The direct internal hits to the cells result in the production of a higher number of mitochondria to overcome the decreased cell function. Under stress, the cellular demand for mitochondrial oxidative metabolism rises. When there is an increased need for tissue energy, it leads to changes in cellular function and cell death eventually influences the severity of the underlying pathology. These changes over time can bring out molecular alterations. ${ }^{7}$ The cellular oxidative phosphorylation process gets affected to a considerable instance. The previous literatures show a recurrence rate of $20 \%$ for oncocytic lesions. According to Bonikos DS et al. the oncocytes form as mitochondrial enzymes decline in number. Other theories of oncocyte formation include Medawar's mutation accumulation hypothesis, Kirkwood's disposable soma theory and the concept of antagonistic pleiotropy. They have described the changes observed could be because of mutation in mitochondrial DNA. Inadequacies involving the cell respiratory chain may not be the constant causative factor as stated by Müller-Höcker et al. One more theory of antagonist pleiotropy reports of mitochondrial senescence in cell differentiation. Sanchez-Cespedes et al. have found a specific mitochondrial genome responsible for the transformed oncocyte. $6-16$

\section{Histopathology and Ultrastructural Studies}

Gross pathology of oncocytoma includes a brown homogenous encapsulated tumour. It is a well-circumscribed lesion consisting chiefly of oncocytes. It displays arrangement of oncocytes in acinar, trabecular or nodular patterns also clear cells can be part of it. Cohen and Batsakis have said that the minor salivary gland oncocytomas are likely to infiltrate the adjacent soft, bone tissues. The cells are polygonal with granular acidophilic cytoplasm, round nucleus and a low nucleus-cytoplasm ratio. The H\&E stained sections display highly eosinophilic shade differences giving rise to dark and light stained oncocytes. They show in bundles with thin strands of fibro-vascular stroma and intraluminal secretions. Presence of oncocytes in normal ducts imply it may not be a part of a tumour always, also contribute to assessing the aetiology behind the origin. They often show extensive cystic change. The phosphotungstic acid haematoxylin (PTAH) staining is useful to exhibit numerous small, dark blue to black granule of mitochondria. Other stains include Luxol fast blue, cresyl violet, thionin and acid-Schiff. Electron microscopy clearly reveals the increased number of mitochondria and their often-swollen peculiar form. The cells have an enlarged spherical stack of mitochondria filling up the $60 \%$ of the cytoplasm. Under scanning electron microscope Riva et al has found oncocytes mitochondria. $4,7,14-17$

constituting

of

pleomorphic

\section{Immunohistochemical Studies}

Antibodies against subunits of the respiratory chain are markers of mitochondrial hyperplasia. Histochemical studies done have found that the oncocytes in the salivary glands and salivary gland tumours have enzymes associated with mitochondria. ${ }^{18}$ As the mitochondria in the oncocytes are increased, the carbonic anhydrase isoenzyme immunoreactivity also increases. The reaction is due to deficient coupling of oxidative phosphorylation. ${ }^{19}$ The heterogeneity in mitochondrial morphology observed in oncocytes is suggestive of an alteration of these organelles. The mitochondrial discrepancies in oncocytes have been documented to justify the hypothesis that the mitochondrial hyperplasia of these cells may be due to a compensatory effect. Oncocytomas show positivity for CK5/6, CK8/18, CK19, EMA. MIB -1 monoclonal antibody was found to stain the cell membrane and cytoplasm of the oncocytes. The salivary gland tumour type-specific markers are very few. Shinmura et al. found that the BSND protein expression part of chloride transport, in the striated duct cells of normal salivary glands besides normal kidney and the inner ear. So BSND immunohistochemistry can be helpful in the diagnosis of the kidney and salivary gland tumours. Ki-67 cell proliferation activity analysis can be useful in planning the treatment for oncocytoma of the salivary gland. ${ }^{20-24}$
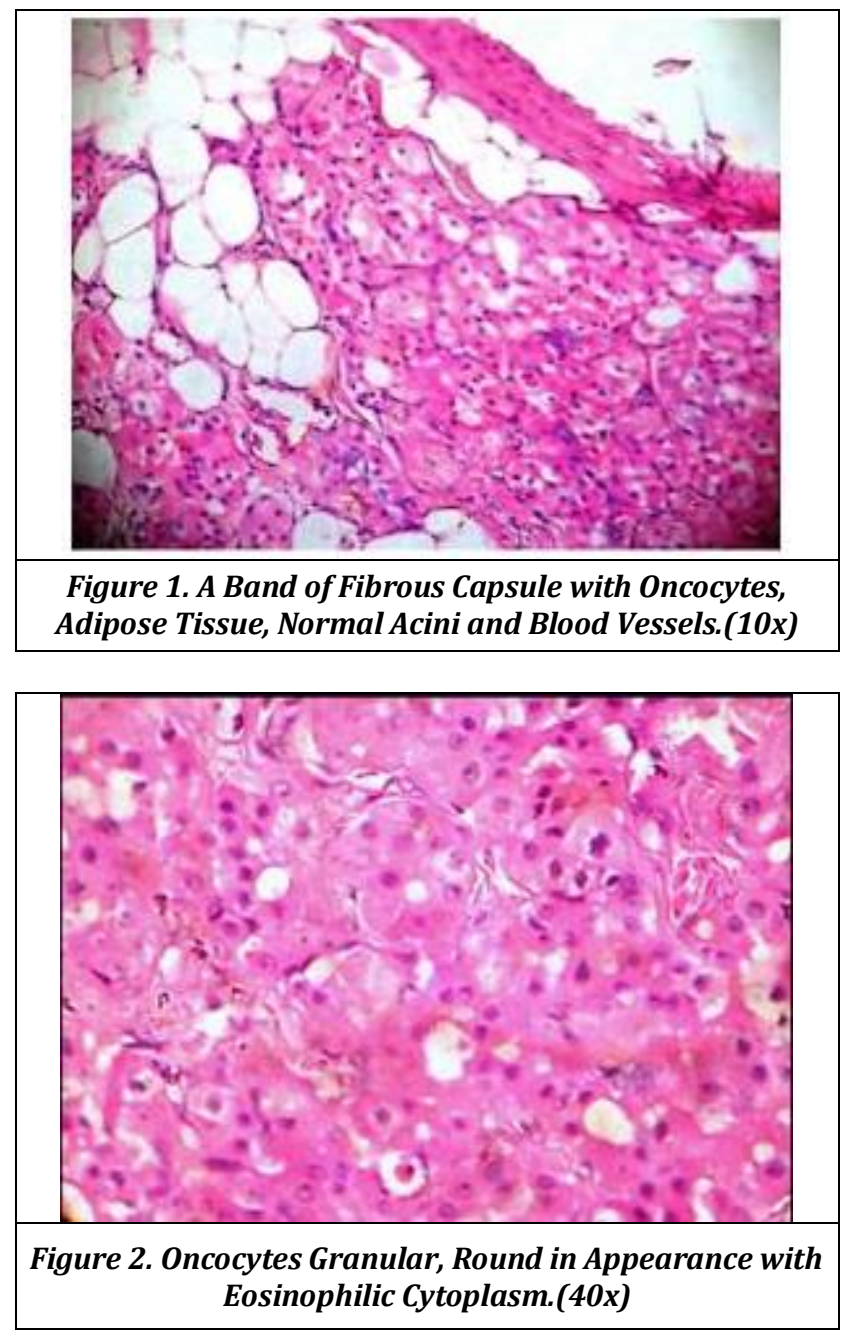


\section{CONCLUSIONS}

Oncocytomas of the salivary glands are generally benign tumours. They rarely show proliferative activity into the adjacent structures. The aetiology behind the formation of these oncocytes are many. Degenerative changes in a cell associated with exposure to drugs or chemicals might cause changes in mitochondria. The mitochondrial DNA point mutations due to altered oxidative phosphorylation complexes are one of the main downsides for the oncocytic genesis. The hypothesis of their origin of transformation has been put forward by several researchers. The role of numerous mitochondria and their functional inability to maintain the bioenergetics pathway forms the basis for these oncocytic changes.

\section{REFERENCES}

[1] Gray SR, Cornog Jr JL, Seo IS. Oncocytic neoplasms of salivary glands: a report of fifteen cases including two malignant oncocytomas. Cancer 1976;38(3):1306-17.

[2] Scott J, Structure and function in aging human salivary glands. Gerodontology 1986;5(3):149-58.

[3] Dardick I, Birek C, Lingen MW, et al. Differentiation and the cytomorphology of salivary gland tumors with specific reference to oncocytic metaplasia. Oral Surgery, Oral Medicine, Oral Pathology, Oral Radiology and Endodontics 1999;88(6):691-701.

[4] Prabakaran SS, Chen F, Aguirre A. Oncocytoma of the parotid gland and its mimickers: a comprehensive review. North American Journal of Medicine and Science 2010;3(4):171-80.

[5] Stomeo F, Meloni F, Bozzo C, et al. Bilateral oncocytoma of the parotid gland. Acta Otolaryngologica 2006;126(3):324-6.

[6] Brandwein MS, Huvos AG. Oncocytic tumors of major salivary glands. A study of 68 cases with follow-up of 44 patients. The American Journal of Surgical Pathology 1991;15(6):514-28.

[7] Capone RB, Ha PK, Westra WH, et al. Oncocytic neoplasms of the parotid gland: a 16-year institutional review. Otolaryngol Head Neck Surg 2002;126(6):65762.

[8] Baum BJ, Ship JA, Wu AJ. Salivary gland function and aging: a model for studying the interaction of aging and systemic disease. Critical Reviews in Oral Biology \& Medicine 1992;4(1):53-64.

[9] Kasper M, Funk RH. Age-related changes in cells and tissues due to advanced glycation end products (AGEs). Archives of Gerontology and Geriatrics 2001;32(3):233-43.

[10] Popovski V, Benedetti A, Monevska DP, et al. Oncocytoma of the deep lobe of the parotid gland. Open access Macedonian Journal of Medical Sciences 2016;;4(2):290-2.
[11] Shellenberger TD, Williams MD, Clayman GL, et al. Parotid gland oncocytosis: CT findings with histopathologic correlation. American Journal of Neuroradiology 2008;29(4):734-6.

[12] Ferreiro JA, Stylopoulos N. Oncocytic differentiation in salivary gland tumours. The Journal of Laryngology \& Otology 1995;109(6):569-71.

[13] Kanazawa H, Furuya T, Murano A, et al. Oncocytoma of an intraoral minor salivary gland: report of a case and review of literature. Journal of Oral and Maxillofacial Surgery 2000;58(8):894-7.

[14] Linnane A, Marzuki S, Ozawa T, et al. Mitochondrial DNA mutations as an important contributor to ageing and degenerative diseases. The Lancet 1989;333(8639):642-5.

[15] Wilkie D, Evans IH, Egilsson V, et al. Mitochondria, cell surface and carcinogenesis. Int Rev Cytol Suppl 1983;15:157-89.

[16] Riva A, Tandler B. Three-dimensional structure of oncocyte mitochondria in human salivary glands: a scanning electron microscope study. Ultrastructural Pathology 2000;24(3):145-50.

[17] Chang A, Harawi SJ. Oncocytes, oncocytosis and oncocytic tumors. Pathol Annu 1992;27(Pt 1):263-304.

[18] Kobayashi H, Hosino MU, Tauchi HI. Ultrastructural study of cytochrome oxidase in oncocytoma. Nagoya J Med Sci 1972;35(1):25-32.

[19] Guaraldi F, Zang G, Dackiw AP, et al. Oncocytic mania: a review of oncocytic lesions throughout the body. Journal of Endocrinological Investigation 2011;34(5):383-94.

[20] Zhou CX, Gao Y. Oncocytoma of the salivary glands: a clinicopathologic and immunohistochemical study. Oral Oncology 2009;45(12):e232-8.

[21] Ito K, Tsukuda M, Kawabe R, et al. Benign and malignant oncocytoma of the salivary glands with an immunohistochemical evaluation of Ki-67. ORL J Otorhinolaryngol Relat Spec 2000;62(6):338-41.

[22] Kuwahara H, Shimazaki M, Kadoya Y, et al. 6 isozymes in oncocytoma and oncocytic epithelial cells. Osaka City Medical Journal 1989;35(2):121-36.

[23] Witt RL, Maygarden SJ. Benign tumors, cyst and tumorlike condition of the salivary glands. In Salivary gland diseases. Surgical and Medical Management 2005: p. 114.

[24] Butow RA, Avadhani NG. Mitochondrial signaling: the retrograde response. Molecular Cell 2004;14(1):1-15. 